\title{
Using electrodiagnostics to define injury patterns amenable to nerve transfer surgery in tetraplegia: an illustrative case report
}

\author{
Elspeth J. R. Hill $\mathbb{D}^{1} \cdot$ Madi El-Haj ${ }^{2} \cdot$ James A. Giles $\mathbb{D}^{3} \cdot$ Ida K. Fox $^{1}$
}

Received: 5 May 2020 / Revised: 29 July 2020 / Accepted: 30 July 2020

(c) International Spinal Cord Society 2020

\begin{abstract}
Introduction Spinal cord injury is a devastating condition affecting a person's independence and quality of life. Nerve transfers are increasingly used to restore critical upper extremity function. Electrodiagnostic studies guide operative planning but the implications for clinical outcomes is not well defined. This case study delineates how clinical examination and electrodiagnostics can define the varying patterns of neuronal injury to guide timing and strategy for optimal outcomes in nerve transfers.

Case presentation We discuss a 20-year-old man with a C6-7 spinal cord injury (SCI). We illustrate how history, physical examination, and electrodiagnostic studies predicted patterns of upper and lower motor neuron injury, confirmed intraoperatively via direct nerve stimulation. We undertook brachialis nerve transfer to the median fascicles supplying flexor digitorum superficialis and anterior interosseous nerve (to restore digit flexion), and supinator nerve transfer to posterior interosseous nerve (to restore digit extension). Preoperative electrodiagnostics of the right upper extremity demonstrated a pure upper motor neuron injury to median innervated muscles, and mixed upper and lower motor neuron injury to radial innervated muscles. These findings were confirmed via intraoperative direct neuromuscular stimulation. The preoperative studies provided important information regarding the anatomic basis and time sensitivity of the proposed nerve transfers. At 2 years post operatively the reconstructed digit flexion and extension resulted in improved hand function and independence.

Discussion Upper and lower motor neuron injuries can coexist in individuals with SCI. This example provides proof-ofconcept that preoperative electrodiagnostic studies predict LMN injury, and surgery can achieve positive outcomes if completed soon after SCI.
\end{abstract}

\section{Introduction}

Nerve transfers have transformed the management of peripheral nerve and brachial plexus injury and have allowed

Supplementary information The online version of this article (https:// doi.org/10.1038/s41394-020-00325-x) contains supplementary material, which is available to authorized users.

Ida K. Fox

foxi@wustl.edu

1 Division of Plastic and Reconstructive Surgery, Washington University School of Medicine, St. Louis, MO, USA

2 Hand and Microvascular Surgery Unit, Hadassah University Hospital, Jerusalem, Israel

3 Department of Neurology, Washington University School of Medicine, St. Louis, MO, USA remarkable restoration of motor function [1,2]. This innovative surgery has been used in people with cervical spinal cord injury (SCI), in whom hand function is essential to undertaking activities of daily living (ADLs), and influences level of independence [3-5]. Nerve transfers offer more fine dexterity, and avoid the prolonged immobilization associated with traditional tendon transfer surgery, as well as broadening the reconstructive options available $[5,6]$.

Nerve transfer in SCI involves coapting an expendable donor nerve above the spinal level of the injury, over which the individual has volitional control, to a nerve within or below the level of injury. Importantly in SCI, a person's weakness may be due to a combination of injury to upper motor neurons (UMN) in the spinal cord's descending motor tracts, and lower motor neuron (LMN) injury at the level of the anterior horn cell. An essential step in the preoperative evaluation is differentiating the pattern of motor neuron injury. This is important because LMN injury 

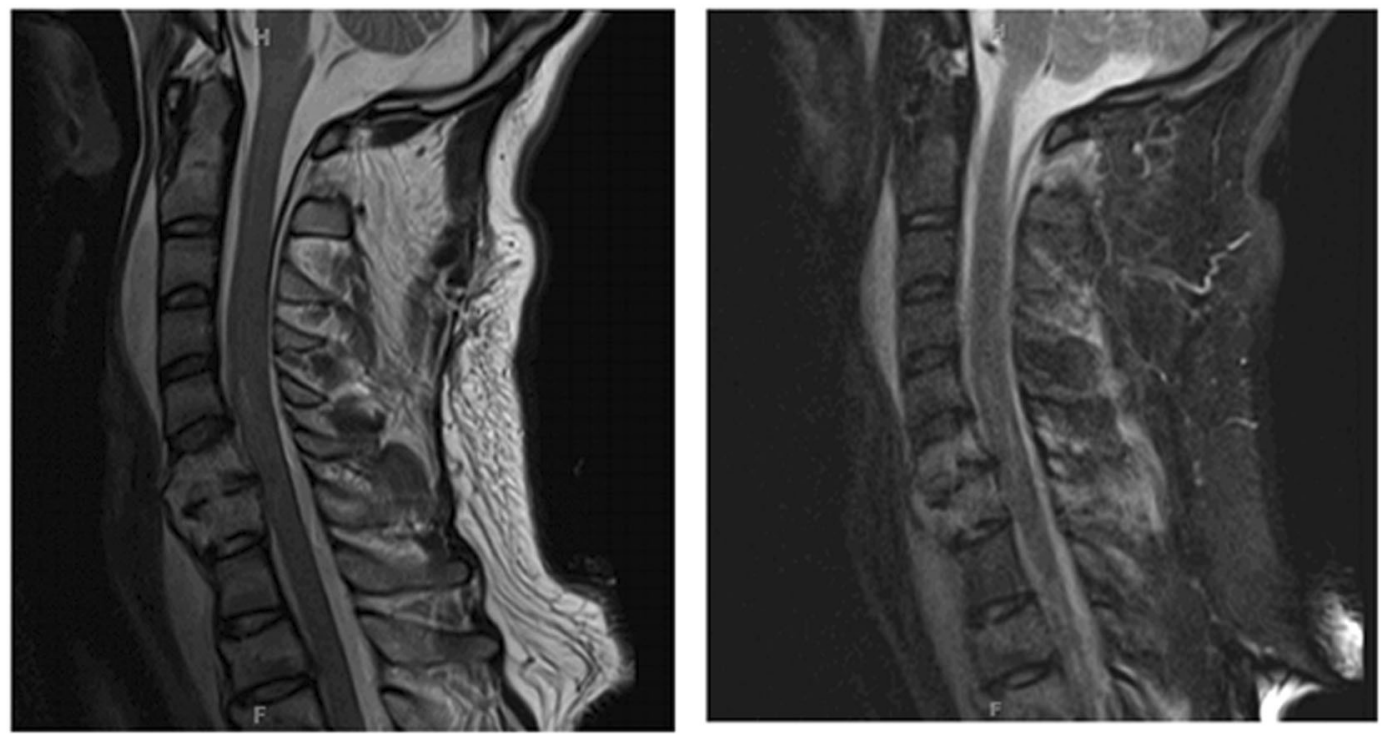

Fig. 1 MRI cervical spine from time of injury. Sagittal T1 (left) and T2 (right) weighted c-spine MRI demonstrating C6-7 comminuted burst fracture with associated spinal cord edema at the C5-7 level.

presupposes a time window in which successful surgery may be undertaken. Irreversible denervation atrophy occurs 12-18 months after a LMN injury, and a nerve transfer will be unsuccessful. This is in contrast to isolated UMN injury, where recipient muscles remain intact [7]. In this situation, the nerve transfer is not time sensitive.

We have previously published our work showing that nerve transfer can successfully restore function in SCI with isolated UMN injury [8]. Others have shown better success in doing early nerve transfer within 12-19 months post SCI [5]. Recently, we published work showing how electrodiagnosis (EDX) can accurately predict the presence or absence of UMN and LMN injury [9].

This clinical case demonstrated how EDX predicts the extent of LMN injury at the level of the recipient nerve and shows how function can be restored in different patterns of UMN and LMN injury if surgery is completed in a timely fashion.

The Washington University Institutional Review Board approved this case review project, and we obtained written informed consent to report these findings. We reviewed the medical records of people with SCI to find a clear illustrative example of the use of EDX studies to aid in operative planning for nerve transfer, and compared preoperative clinical examination and EDX findings to recorded values from intraoperative nerve stimulation. To illustrate the learning points from the case, we developed a narrative review of how preoperative EDX, intraoperative neuromuscular stimulation and timing of surgery were used to develop an individualized treatment strategy, and present postoperative outcomes. All applicable institutional and governmental regulations concerning the ethical use of human volunteers were followed during the course of this research.

\section{Case presentation}

A 20-year-old man presented eight months after sustaining a cervical SCI. His injury was American Spinal Injury Association $\mathrm{A}$ at the $\mathrm{C} 7$ level (Fig. 1). He remained dependent on others for ADL, including intermittent straight catheterization, and used an electric wheelchair. $\mathrm{He}$ reported no gains or changes in function for 6 months prior to presentation. His goal was to regain the ability to selfcatheterize and to independently feed himself.

Physical examination revealed absent motor function in his bilateral hands, relying on tenodesis alone to handle objects. Detailed upper extremity motor examination results are in Table 1.

Preoperative EDX were performed. Findings were consistent with a mixed pattern of UMN and LMN injury at the C7-T1 levels. There were normal median, reduced ulnar and absent radial compound muscle action potentials (CMAPs), indicating mixed UMN and LMN injury; the intact median nerve CMAP indicated an UMN injury with functioning peripheral nerve, whereas the absent radial nerve CMAP indicated UMN and LMN injury. There were preserved sensory nerve action potentials (SNAPs) indicating intact dorsal root ganglia and distal sensory nerves. Further, there was electromyogram (EMG) evidence of variable denervation and absent motor unit potentials at the C7-T1 myotomes (Table 2).

A surgical plan was formed based on the individual's goals, his exam and the EDX (Fig. 2). Surgery was completed under non-paralytic general anesthesia and intraoperative nerve stimulation was undertaken to confirm the functional status of recipient nerves. 
Table 1 Upper extremity examination.

\begin{tabular}{|c|c|c|c|}
\hline Region & Muscle & Right & Left \\
\hline \multirow[t]{5}{*}{ Shoulder/upper arm } & Deltoid & 5 & 5 \\
\hline & Biceps & 5 & 5 \\
\hline & Brachialis & 5 & 5 \\
\hline & Brachioradialis & 5 & 5 \\
\hline & Triceps & 4 & 4 \\
\hline \multirow[t]{2}{*}{ Forearm } & Pronation & 5 & 5 \\
\hline & Supination & 5 & 5 \\
\hline \multirow[t]{3}{*}{ Wrist extension } & Extensor carpi radialis longus & 5 & 5 \\
\hline & Extensor carpi radialis brevis & 5 & 5 \\
\hline & Extensor carpi ulnaris & 0 & 0 \\
\hline \multirow[t]{3}{*}{ Wrist flexion } & Flexor carpi radialis & 5 & 3 \\
\hline & Flexor carpi ulnaris & 0 & 0 \\
\hline & Palmaris & 0 & 0 \\
\hline \multirow[t]{4}{*}{ Finger extension } & Extensor pollicis longus & 0 & 0 \\
\hline & Extensor indicis proprius & 0 & 0 \\
\hline & Extensor digitorum communis & 0 & 0 \\
\hline & Extensor digiti minimi & 0 & 0 \\
\hline \multirow[t]{3}{*}{ Finger flexion } & Flexor pollicis longus & 0 & 0 \\
\hline & Flexor digitorum profundus & 0 & 0 \\
\hline & Flexor digitorum superficialis & 0 & 0 \\
\hline \multirow[t]{6}{*}{ Intrinsic function } & Thenars & 0 & 0 \\
\hline & Adductor pollicis & 0 & 0 \\
\hline & Hypothenar & 0 & 0 \\
\hline & Lumbricals & 0 & 0 \\
\hline & Dorsal interossei & 0 & 0 \\
\hline & Palmar interossei & 0 & 0 \\
\hline
\end{tabular}

Detailed preoperative results of manual muscle testing using Medical Research Council (MRC) scale.

\section{Brachialis nerve to anterior interosseous nerve (AIN) and flexor digitorum superficialis (FDS) nerve transfer to restore digit flexion}

Intraoperative stimulation of brachialis branches showed excellent motor response. Intraoperative stimulation of the median nerve motor fascicles produced contraction of flexor pollicis longus (FPL), flexor digitorum profundus (FDP) to index and long finger, and FDS. A brachialis nerve transfer to both the AIN and FDS nerve fascicles of the median nerve was undertaken (Fig. 3) (Video 1S).

\section{Supinator nerve to posterior interosseous nerve (PIN) transfer to restore digit extension}

Intraoperative stimulation of donor showed a strong response to the supinator muscle. Intraoperative stimulation of the PIN induced very weak thumb extension via extensor pollicis longus (EPL) only and no stimulation of the extensor digitorum comminis (EDC). A supinator to PIN transfer was undertaken to restore hand opening (Fig. 4) (Video 2S).

\section{Outcomes}

Postoperatively there was no downgrade in donor muscle function. At 2 years post operatively recipient muscle EDC power increased from MRC 0 to 4 and FPL from 0 to 2 (Table 3 and Video 3S) without change in FDS power. Functionally he achieved his goal of no longer needing assistance to feed himself (he required a suprapubic catheter in the interim, so did not achieve his self-catheterization goals). Although his nerve transfers restored only small increases in strength (M2 FPL and M4-for EDC-Video $3 \mathrm{~S}$ ), in the spinal cord injured person modest motor gains can translate to significant functional gains by augmenting tenodesis and existing function. Based on this individual's preferences, nerve transfers were completed instead of the less dextrous but more powerful traditional tendon transfer options $[2,5]$.

\section{Discussion}

Here, we present a case of nerve transfer as a surgical technique to restore upper extremity function in a person with a mixed pattern of UMN and LMN cervical SCI. We focus the discussion on how preoperative EDX may be used as to predict intraoperative nerve stimulation in order to develop a pathophysiologic and anatomic basis, as well as a rational timeline, for nerve transfers in such individuals.

The preoperative evaluation of the recipient and the donor nerves in SCI presents unique challenges. There is variation in the pattern of neurologic injury for a given pattern of impairment, and it can be difficult to discern whether weakness is due to injury of UMN, anterior horn cell, or a more distal superimposed peripheral nerve injury [7]. Hence, a multimodal approach to the clinical examination and preoperative planning is advisable [10]. The role for EDX in preoperative evaluation of nerve transfer has been more clearly defined in brachial plexus and peripheral nerve injury [11, 12]. EDX comprises both nerve conduction studies (NCS) and electromyography (EMG). EMG is a qualitative tool and in SCI it has limited utility to predict the type and degree of motor neuron injury, as mixed UMN and LMN patterns of injury coexist. Furthermore, extensive denervation atrophy in those with long-standing SCI can distort anatomic landmarks, making EMG challenging. By contrast, the CMAP amplitude measured in NCS adds quantitative information about the numbers of nerve fibers within the tested nerve segment. Our case example shows how the CMAP amplitudes of the distal median (normal) and radial nerve (absent) segments correlated well with the 
Table 2 Preoperative electrodiagnostic testing.

\begin{tabular}{|c|c|c|c|c|c|c|}
\hline \multicolumn{7}{|c|}{ Motor nerve conduction studies } \\
\hline Nerve/site & Amplitude (mV) & Latency (ms) & Segments & Distance (mm) & Lat. Diff. (ms) & Velocity $\left(\mathrm{ms}^{-1}\right)$ \\
\hline \multicolumn{7}{|c|}{ R Median-APB } \\
\hline Wrist & 9.0 & 3.23 & Wrist-APB & 70 & & \\
\hline Elbow & 9.0 & 7.29 & Elbow-wrist & 215 & 4.06 & 52.9 \\
\hline \multicolumn{7}{|l|}{ R Ulnar-FDI } \\
\hline Wrist & 2.1 & 3.96 & Wrist-FDI & & & \\
\hline Below elbow & 1.9 & 7.34 & Below elbow-wrist & 200 & 3.39 & 59.1 \\
\hline Above elbow & 1.1 & 9.06 & Above elbow-below elbow & 87 & 1.72 & 50.6 \\
\hline \multicolumn{7}{|c|}{ R Radial-EIP } \\
\hline Forearm & NR & NR & Forearm-EIP & & & \\
\hline
\end{tabular}

Sensory nerve conduction studies

Nerve/site Amplitude $(\mu \mathrm{V}) \quad$ Onset latency $(\mathrm{ms}) \quad$ Peak latency $(\mathrm{ms}) \quad$ Onset latency (ms) Distance $(\mathrm{mm}) \quad$ Velocity $\left(\mathrm{ms} \mathrm{s}^{-1}\right)$

R Median, Ulnar-Digits 1, 3, 5

Median digit $131 \quad 2$.

Median digit 322

Ulnar digit $5 \quad 20 \quad 2.9$

$2.2 \quad 2.8$

$3.0 \quad 3.0$

2.0

R Radial-anatomical snuffbox (forearm)

Forearm 19

Electromyography (EMG)

Muscle

Insertional

Spontaneous

Motor unit action potentials

\begin{tabular}{|c|c|c|c|c|c|c|c|c|}
\hline R FDS (median) & Activity & Fib. & $\begin{array}{l}\text { PSW } \\
\text { None }\end{array}$ & $\begin{array}{l}\text { Fasc. } \\
\text { None }\end{array}$ & Dur. & Amp. & Poly. & $\begin{array}{l}\text { Recruit. } \\
\text { None }\end{array}$ \\
\hline R EDC (radial) & Increased & $2+$ & $2+$ & None & & & & None \\
\hline
\end{tabular}

Results of the preoperative nerve conduction studies (NCS) and electromyography (EMG) are presented. Note that the amplitude for the motor NCS across specific tested segments varies for the median, ulnar and radial nerve. For the median nerve, we focus on the amplitude for the wrist to $\mathrm{ABP}$ segment as this provides surrogate information about the number of nerve fibers at the C8/T1 level and suggests continuity of the lower motor neurons at this level. Although specific testing of the recipient AIN segment may be theoretically possible, this surrogate testing is reliable, easier to perform and, as illustrated by the intraoperative stimulation, correlates well to the wrist to APB segment NCS amplitude. For the radial nerve, we test the forearm to EIP segment, which provides direct information about the number of nerve fibers in the recipient PIN. Note that the EMG results provide only qualitative information about denervation (EDC shows active denervation as indicated by the presence of fibs and PSW's). The absence of motor unit action potentials further supports the presence of an upper motor neurons injury.

Lat. Diff. latency difference, $A P B$ abductor pollicis brevis, FDI first dorsal interosseous, EIP extensor indicis proprius, Onset Diff. onset difference, Fib. fibrillation, PSW positive sharp wave, Fasc. fasciculation, Dur. duration, Amp. amplitude, Poly. polyphasia, Recruit. recruitment, Activ. activation, $F D S$ flexor digitorum duperficialis, $A D M$ abductor digiti minimi, $E D C$ - extensor digitorum communis.

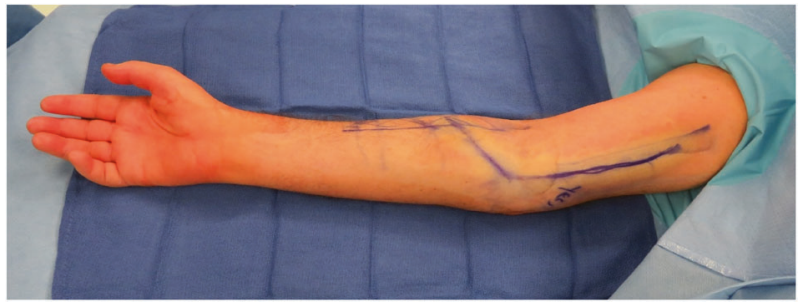

Fig. 2 Preoperative markings. Upper arm: Proximal medial incision is made at the distal third of the arm between the biceps and brachialis muscle bellies, to directly approach the brachialis branches of the musculocutaneous nerve. Forearm: proximal lateral curvilinear incision is made over the radial tunnel (Reprinted with permission. Copyright 2018, nervesurgery.wustl.edu). presence or absence of intraoperative neuromuscular stimulation of the corresponding musculature. This finding is supported by a recent study correlating normal CMAP amplitudes with the presence of neuromuscular intraoperative stimulation [9].

We used preoperative EDX to determine the pattern of motor neuron injury in our intended recipient nerves. The EDX of the right median nerve showed an intact CMAP and SNAP with absent activation of the FDS muscle and no fibrillations or positive sharp waves on EMG. This pattern was consistent with a purely UMN injury to the descending corticospinal tract supplying the anterior horn cell bodies of the median nerve branch to FDS; there was an absence of 


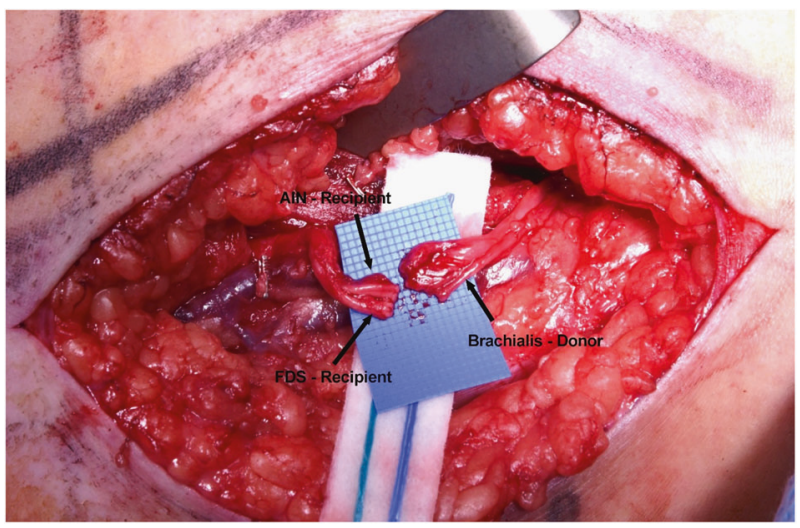

Fig. 3 Brachialis nerve to AIN and FDS nerve transfer. Figure demonstrates the donor brachialis branches of the musculocutaneous nerve, and the recipient FDS and AIN fascicles of the median nerve. Intraoperative measurement revealed $14-16 \mathrm{~cm}$ of nerve regeneration required from coaptation to target muscle re-innervation (Reprinted with permission. Copyright 2018, nervesurgery.wustl.edu).

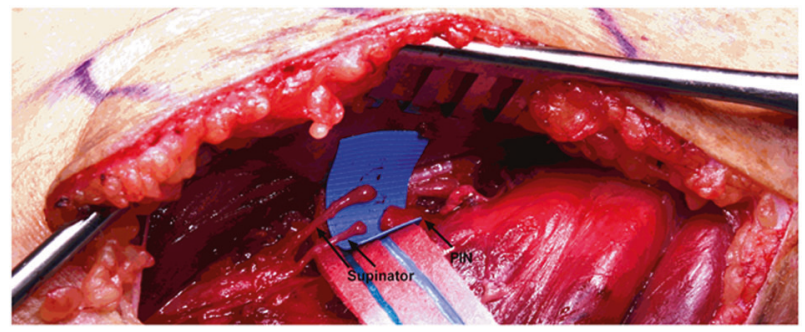

Fig. 4 Supinator nerve to PIN transfer. Figure demonstrates proximally the donor supinator nerve and distally the recipient PIN. Intraoperative measurement revealed $2-4 \mathrm{~cm}$ of never regeneration required to reach target muscle (Reprinted with permission. Copyright 2018, nervesurgery.wustl.edu).

Table 3 Pre- and post-operative motor outcomes.

\begin{tabular}{llllll}
\hline & Pre-Op & & \multicolumn{2}{l}{2 years } \\
\cline { 2 - 3 } \cline { 5 - 6 } & Right & Left & & Right & Left \\
\hline Anterior deltoid & 5 & 5 & 5 & 5 \\
Elbow flexors & 5 & 5 & 5 & 5 \\
Elbow extensors & 4 & 4 & 5 & 4 \\
Wrist extensors & 5 & 5 & 5 & 5 \\
Extensor digitorum & 0 & 0 & 4 & 0 \\
Opponens pollicis & 0 & 0 & 0 & 0 \\
Flexor pollicis longus & 0 & 0 & 2 & 0 \\
Finger flexors & 0 & 0 & 0 & 0 \\
Finger abductors & 0 & 0 & 0 & 0 \\
First dorsal interosseus & 0 & 0 & 0 & 0 \\
\hline
\end{tabular}

Preoperative and 2 years post operative outcomes of motor testing using the MRC scale.

UMN voluntary control with a normal LMN. This was corroborated via intraoperative neuromuscular stimulation, whereby the median nerve showed excellent motor responses. Outcomes were acceptable to this individual with a gain in FPL function that augmented tenodesis (additional tendon transfer surgery was offered but he declined).

In contrast, the study of the right radial nerve showed an absent CMAP with intact SNAP, with absent activation of the EDC and moderate fibrillations and positive sharp waves on EMG. This pattern was consistent with a mixed UMN and LMN injury. Again, the lack of voluntary control (absent activation on EMG) indicated an injured corticospinal tract. However, this time there was evidence of motor neuropathy and neurogenic muscle changes in the absence of a sensory injury, which is consistent with a concomitant LMN injury at the level of the anterior horn cells within the spinal cord. Again, the intraoperative neuromuscular stimulation correlated with preoperative testing, whereby the radial nerve showed only a very weak EPL and no EDC response. In this individual, the supinator nerve to PIN nerve transfer was completed within eight months of the SCI, enabling the nerve regeneration to reach the target muscle before terminal denervation atrophy occurred, resulting in improved finger extension.

This case demonstrates how EDX, and specifically how quantitative NCS data, may be used to predict intraoperative nerve stimulation when planning nerve transfers, and should form part of a comprehensive preoperative assessment for nerve transfer in individuals with SCI in whom complex, mixed patterns of injury are common. This example provides proof-of-concept that preoperative EDX predicts LMN injury and positive clinical results can be achieved if surgery is completed in this injury pattern soon after SCI. Additional study correlating preoperative EDX and timing of surgery with clinical outcomes will provide further support to our case report findings.

Funding This study was funded by Craig H. Neilsen Foundation for Spinal Cord Injury Research on the Translation Spectrum (SCIRTS) Grant: "Nerve Transfers to Restore Hand Function in Cervical Spinal Cord Injury".

\section{Compliance with ethical standards}

Conflict of interest The authors declare that they have no conflict of interest.

Ethical approval This study was approved by our Institutional Review Board. This IRB-approved study was conducted in accordance with the ethical standards of the responsible committee on human experimentation (institutional and national) and with the Helsinki Declaration of 1975 , as revised in 2008 .

Informed consent Informed consent, as well as any necessary Health Insurance Portability and Accountability Act consent was obtained in accordance with IRB approvals.

Publisher's note Springer Nature remains neutral with regard to jurisdictional claims in published maps and institutional affiliations. 


\section{References}

1. Boyd KU, Nimigan AS, Mackinnon SE. Nerve reconstruction in the hand and upper extremity. Clin Plast Surg. 2011;38:643-60.

2. Hill EJR, Fox IK. Current best peripheral nerve transfers for spinal cord injury. Plast Reconstr Surg. 2019;143:184e-198e.

3. Bertelli JA, Tacca CP, Ghizoni MF, Kechele PR, Santos MA. Transfer of supinator motor branches to the posterior interosseous nerve to reconstruct thumb and finger extension in tetraplegia: case report. J Hand Surg Am. 2010;35:1647-51.

4. Bertelli JA, Ghizoni MF. Nerve transfers for elbow and finger extension reconstruction in midcervical spinal cord injuries. $\mathbf{J}$ Neurosurg. 2015;122:121-7.

5. van Zyl N, Hill B, Cooper CA, Hahn JB, Galea MP. Nerve transfers for the restoration of upper limb function in tetraplegia: expanding on traditional tendon-based techniques in a prospective case series. Lancet. 2019;394:565-75.

6. Ray WZ, Mackinnon SE. Clinical outcomes following median to radial nerve transfers. J Hand Surg Am. 2011;36:201-8.
7. Coulet B, Allieu Y, Chammas M. Injured metamere and functional surgery of the tetraplegic upper limb. Hand Clin. 2002;18:399-412.

8. Fox IK, Novak CB, Kahn LC, Mackinnon SE, Ruvinskaya R, Juknis N. Using nerve transfer to restore prehension and grasp 12 years following spinal cord injury: a case report. Spinal Cord Ser Cases. 2018;4:37.

9. Jain NS, Hill EJR, Zaidman CM, Novak CB, Hunter DA, Juknis $\mathrm{N}$, et al. Evaluation for late nerve transfer surgery in spinal cord injury: predicting the degree of lower motor neuron injury. J Hand Surg Am. 2020;45:95-103.

10. Fox IK, Novak CB, Krauss EM, Hoben GM, Zaidman CM, Ruvinskaya $\mathrm{R}$, et al. The use of nerve transfers to restore upper extremity function in cervical spinal cord injury. PM\&R. 2018;10:1173-1184.e2.

11. Noland SS, Bishop AT, Spinner RJ, Shin AY. Adult traumatic brachial plexus injuries. J Am Acad Orthop Surg. 2019;27:705-16.

12. Schreiber JJ, Feinberg JH, Byun DJ, Lee SK, Wolfe SW. Preoperative donor nerve electromyography as a predictor of nerve transfer outcomes. J Hand Surg Am. 2014;39:42-49. 